\title{
Wetting on nanorough surfaces
}

\author{
T. Biben and L. Joly \\ Université de Lyon, F-69000, France; Univ. Lyon 1, \\ Laboratoire PMCN; CNRS, UMR 5586; F-69622 Villeurbanne Cedex
}

\begin{abstract}
We present in this letter a free-energy approach to the dynamics of a fluid near a nano-structured surface. The model accounts both for the static phase equilibrium in the vicinity of the surface (wetting angles, Cassie-Wenzel transition) and the dynamical properties like liquid slippage at the boundary. This method bridges the gap between phenomenological phase-field approaches and more macroscopic lattice-Boltzmann models.
\end{abstract}

Viscous dissipation is a major problem in micro or even nanofluidic systems [1]. Large pressure gradients are indeed necessary to produce a significant flow at small scale. This effect is well illustrated by the Poiseuille law relating the flux to the size of the duct: for a cylindrical duct of radius $R$, one can check that the flux decreases like $R^{4}$ when a given pressure gradient is applied. The Poiseuille law is however obtained by assuming no-slip boundary conditions (BC) for the liquid at the walls, and recent experiments have shown that this might not always be the case [2]. In the presence of partial slip BC, one can expect the situation to be less penalizing, specially if the slip length associated to the boundary is of the order of the duct radius or even larger [3]. Superhydrophobic surfaces have thus attracted a considerable interest recently as potential candidates for highly slipping surfaces: if the liquid is repelled by the surface, one can imagine that a thin layer of gas (air or vapor) at the surface could produce a lubricating effect. The situation is however more complex since natural or artificial superhydrophobic surfaces are extremely rough: roughness is indeed a key ingredient in superhydrophobicity, since it favors the trapping of air or vapor bubbles at the boundaries [4]. This effect is due to capillarity, and the question to understand the coupling between the three ingredients, hydrodynamics, capillarity and surface roughness is a true challenge. From the experimental point of view, the situation is still very controversial, with measured slip lengths varying on several orders of magnitude [3]. It is thus crucial to have numerical models able to investigate the static and the dynamic properties of a fluid at a structured boundary.

Several approaches have been considered (see [3] for a recent review), ranging from molecular dynamics at the nanometric scales [5] to hydrodynamic models (including the lattice-Boltzmann approach [6]) at micronic scales [7, 8, 9, 10, 11, 12], and a very recent phase-field model at intermediate scales [13]. The method we shall consider in this letter is intermediate between phasefield phenomenological approaches, not accounting for the specificities of the gas phase (low viscosity and high compressibility) and the more macroscopic lattice Boltzmann approaches. It accounts explicitly for a possible liquid-vapor coexistence in the vicinity of a structured surface, and also accounts for an hydrodynamic flow with liquid slippage at the surface. This model is able to re- produce both static properties such as wetting angles or Cassie-Wenzel states, but also to predict the effective slip lengths of structured interfaces by accounting explicitly for the low viscosity gas layer that forms at the boundary. Moreover, this approach gives access to the intrusion dynamics of the fluid inside the pores of the structured surface, which is an important phenomenon when the liquid is submitted to large pressures.

The model is constructed as follows:

$$
\frac{\partial \rho}{\partial t}+\nabla \cdot(\rho \boldsymbol{v})=-C\left(\frac{\delta \Omega}{\delta \rho}\right) \text { or }=C^{\prime} \Delta\left(\frac{\delta \Omega}{\delta \rho}\right)
$$

is the transport equation for the local density field $\rho(\boldsymbol{r}, t)$, where $t$ is time and $\boldsymbol{r}$ denotes the position; $\boldsymbol{v}(\boldsymbol{r}, t)$ is the local velocity field and $\Omega[\rho]=F[\rho]-\mu \int \rho(\boldsymbol{r}) \mathrm{d} \boldsymbol{r}$ is the grand-potential functional associated to the thermodynamic equilibrium between the liquid and the vapor phase $(F[\rho]$ is the free-energy). $\rho$ is thus a physical order parameter here, and not simply a mathematical object to identify the phases as in usual phase-field approaches [13]. The convective transport of the density field is included in the left term, while the local thermodynamic equilibrium is contained in the right terms: the first writing corresponds to the Allen-Cahn model [14], interesting for the study of the phase diagram (non-conserved dynamics, fixed chemical potential $\mu$ ), and the second writting is the Cahn-Hilliard model [15], corresponding to a more realistic conserved dynamics, that we shall use to investigate the non-equilibrium properties. $C^{\prime}$ is related to the molecular diffusion constant $D$ in a bulk phase by the relation: $D=C^{\prime} f$ " $(\rho)$ where $f$ " denotes the second derivative of the bulk free energy $f(\rho)$ at the bulk density $\rho$. In this work we used $D=2300 \mu \mathrm{m}^{2} / \mathrm{s}$ in the liquid phase, to match the auto-diffusion constant in pure water $\left(D=2270 \mu \mathrm{m}^{2} / \mathrm{s}\right)$. The free energy functional is $F[\rho]=\int \mathrm{d} \boldsymbol{r}\left\{f(\rho)+\frac{w^{2}}{2}|\nabla \rho|^{2}+\rho V_{\text {wall }}(\boldsymbol{r})\right\}$, with $f(\rho)=k_{\mathrm{B}} T \rho\left\{\log \left(\frac{\rho b}{1-\rho b}\right)-1\right\}-a \rho^{2}$ the free energy density of the bulk van der Waals theory ( $a$ is the mean field attractive energy between two atoms, and $b$ is the close packing inverse density). The square gradient term accounts for the natural thickness of the liquid-vapor interface and gives rise to the surface tension $\gamma_{\mathrm{LV}}=w \int_{\rho_{\mathrm{V}}}^{\rho_{\mathrm{L}}} \sqrt{2(f(\rho)-\mu \rho+P)} \mathrm{d} \rho$ [16] where $P$ is the bulk pressure, $\rho_{\mathrm{L}}$ and $\rho_{\mathrm{V}}$ are the coexisting liquid and vapor densities respectively. $w$ is related to the in- 
terfacial thickness. $V_{\text {wall }}(\boldsymbol{r})$ is the interaction potential applied by the wall. Equation (1) has to be solved with the corresponding equation for the velocity field:

$$
\frac{\partial \rho_{\mathrm{m}} \boldsymbol{v}}{\partial t}+\nabla \cdot\left(\rho_{\mathrm{m}} \boldsymbol{v} \boldsymbol{v}\right)=\nabla \cdot \boldsymbol{\sigma}-\rho \nabla\left(\frac{\delta F[\rho]}{\delta \rho}\right)+\boldsymbol{f}_{\mathrm{wall}},
$$

where $\rho_{\mathrm{m}}(\boldsymbol{r}, t)$ is the local mass density $\left(\rho_{\mathrm{m}}(\boldsymbol{r}, t)=\right.$ $M \rho(\boldsymbol{r}, t)$, with $M$ the molecular mass $), \boldsymbol{\sigma}=\eta(\rho)(\nabla \boldsymbol{v}+$ $\nabla \boldsymbol{v}^{t}$ ) is the local viscous stress tensor (with $\eta(\rho)$ the shear viscosity). Although more accurate prescriptions can be considered, we use in this work the simple ansatz $\eta(\rho)=\rho_{\mathrm{m}} \nu$, where the kinematic viscosity $\nu$ is assumed to be the same in the liquid and the vapor phase. The second term $-\rho \nabla\left(\frac{\delta F[\rho]}{\delta \rho}\right)$ is the thermodynamic force field applied by the density field $\rho(\boldsymbol{r})$ to the flow. This term contains both the compressibility of the fluid, fixed by the van der Waals theory, and the capillary force when interfaces are present. The last term of equation (2) accounts for the interaction with the wall.

We shall now discuss the interactions between the fluid and the wall. The two fields $\rho(\boldsymbol{r}, t)$ and $\boldsymbol{v}(\boldsymbol{r}, t)$ are defined in the whole parallelepipedic resolution box, and are thus defined and solved inside the walls. While the static properties of the wall are controlled by $V_{\text {wall }}(\boldsymbol{r})$ (expulsion of the fluid from the wall and wetting properties), the boundary conditions for the velocity field are controlled by $\boldsymbol{f}_{\text {wall }}$. A prerequisite for a correct description of the dynamical behavior of the liquid at superhydrophobic surfaces is to model properly the intrinsic slip properties of the liquid on a flat solid surface. Our prescription for $\boldsymbol{f}_{\text {wall }}$ ensures partial slip BC in this case. These boundary conditions correspond to the continuity of the tangential stress $\sigma_{\| z}$ at the fluid/solid interface: $\sigma_{\| z}$ in the fluid equals $-\lambda v_{\|}\left(z_{\mathrm{s}}\right)$, the friction stress applied by the wall, where $z$ is the normal direction, $\|$ the tangential direction under consideration, $\lambda$ is the friction coefficient and $v_{\|}\left(z_{\mathrm{s}}\right)$ the slip velocity at the wall $\left(z=z_{\mathrm{s}}\right)$. The partial slip BC thus expresses:

$$
v_{\|}\left(z_{\mathrm{s}}\right)=\frac{1}{\lambda} \sigma_{\| z}=\left.\frac{\eta}{\lambda} \frac{\partial v_{\|}}{\partial z}\right|_{z=z_{\mathrm{s}}}=\left.b \frac{\partial v_{\|}}{\partial z}\right|_{z=z_{\mathrm{s}}},
$$

where $b=\eta / \lambda$ is the slip length, and $\eta$ is the shear viscosity of the fluid. If $b, \eta$ and the slip velocity are known, the stress at the interface is $-\eta v_{\|}\left(z_{\mathrm{s}}\right) / b$.

The bare interaction between an atom of the wall and an atom of the fluid is the Lennard-Jones potential in our model: $V_{\mathrm{LJ}}(r)=4 \epsilon\left[(\sigma / r)^{12}-(\sigma / r)^{6}\right]$. The global potential applied by the wall on a single atom of the fluid is $V_{\text {wall }}(\boldsymbol{r})=\int \rho_{\text {wall }}\left(\boldsymbol{r}^{\prime}\right) V\left(\left|\boldsymbol{r}^{\prime}-\boldsymbol{r}\right|\right) \mathrm{d} \boldsymbol{r}^{\prime}$, where $\rho_{\text {wall }}(\boldsymbol{r})$ is the density of atoms in the wall (assumed to be a constant inside the wall and zero elsewhere), and $V(r)$ is the bare interaction potential $\left[V_{\mathrm{LJ}}(r)\right]$ multiplied by the pair distribution function between an atom of the wall and an atom of the fluid. The main property of this pair distribution function is to vanish when $r \rightarrow 0$ since two atoms cannot overlap, while $V_{\mathrm{LJ}}(r) \rightarrow+\infty$ in this

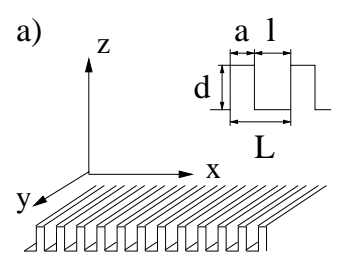

c)

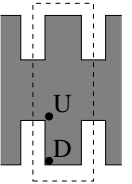

Wenzel

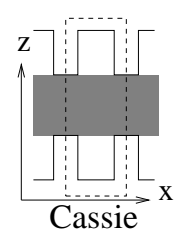

d)

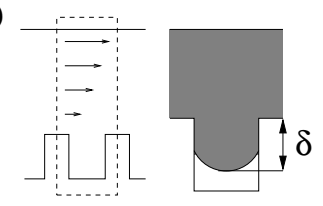

Figure 1: Schematic views of the systems under consideration.

limit. The product of these two functions thus goes to a well defined limit in $r=0$. As a result, the potential energy inside the wall is large, but finite. We shall denote by $V_{\text {cut }}$ this energy and use the simple ansatz: $V_{\text {wall }}(\boldsymbol{r})=\min \left(V_{\text {cut }}, \int \rho_{\text {wall }}\left(\boldsymbol{r}^{\prime}\right) V_{\mathrm{LJ}}\left(\left|\boldsymbol{r}^{\prime}-\boldsymbol{r}\right|\right) \mathrm{d} \boldsymbol{r}^{\prime}\right)$. This prescription fixes the interaction to be of the Lennard-Jones type close to the boundary with the fluid, and to be $V_{\text {cut }}$ inside the wall. $V_{\text {cut }}$ fixes the value of the fluid density inside the wall, chosen to be negligible as compared to the density of the vapor phase $\left(\rho \sigma^{3} \lesssim 10^{-3}\right)$. In this limit, the results become independent of $V_{\text {cut }}$.

The force field $\boldsymbol{f}_{\text {wall }}$ exerted by the wall on the fluid is chosen as a friction force:

$$
\boldsymbol{f}_{\text {wall }}=-k \epsilon \rho(\boldsymbol{r}) \boldsymbol{v}(\boldsymbol{r}) \int \mathrm{d} \boldsymbol{r}^{\prime}\left\{\rho_{\text {wall }}\left(\boldsymbol{r}^{\prime}\right) \mathrm{e}^{-\frac{3}{2} \frac{\left(\boldsymbol{r}^{\prime}-\boldsymbol{r}\right)^{2}}{\sigma^{2}}}\right\}
$$

. This force is proportional to the interaction energy $\epsilon$ between the solid and the fluid [3, 17]. This prescription enabled us to reproduce correctly the evolution of the BC with the wetting properties. In particular, we obtain a transition from a no-slip BC in a wetting situation $(\theta \sim$ $0^{\circ}$ ) to a partial slip BC with $b \sim 10 \mathrm{~nm}$ (depending on the value of $k)$ in a non-wetting situation $\left(\theta \sim 120^{\circ}\right)$.

The two equations (11) and (2) can be solved numerically on a regular lattice (cubic unit cell in 3D or square in $2 \mathrm{D}$ ) with periodic $\mathrm{BC}$ at the edges of the resolution box on the appropriate quantities, as we shall discuss. The wall is defined in the resolution box by the function $\rho_{\text {wall }}(\boldsymbol{r})$. We consider a slab geometry corresponding to a fluid confined between two walls, the bottom wall is textured with a periodic structure (crenels in this study), while the top wall can either be structured in static studies (Fig 1 1 c), or planar in dynamic situations (Fig 1 - d). The density $\rho(\boldsymbol{r})$ is periodic in the slab (x-y) direction, but also in the $\mathrm{z}$ direction since it goes to a constant value (nearly vanishing) inside the walls. The velocity field is periodic in the $\mathrm{x}-\mathrm{y}$ direction, but not in the $\mathrm{z}$ direction where a linear shear $\boldsymbol{v}_{\mathrm{s}}(\boldsymbol{r})$ is applied. In this case, the periodic BC is taken on $\boldsymbol{u}(\boldsymbol{r})=\boldsymbol{v}(\boldsymbol{r})-\boldsymbol{v}_{\mathrm{s}}(\boldsymbol{r})$ in any directions. By using a simple Euler scheme, equations (10) and (2) can be solved iteratively starting from an initial configuration for $\rho(\boldsymbol{r})$ and $\boldsymbol{v}(\boldsymbol{r})$. Taking advantage of the 
periodicity of $\rho(\boldsymbol{r})$ and $\boldsymbol{u}(\boldsymbol{r})$, we use an implicit method in the Fourier space to improve the numerical stability, but simpler schemes can be used as well.

To illustrate the ability of the model to account for the small scale physics of the interfaces, we consider two situations. The first study corresponds to a static case, where $\boldsymbol{v}_{\mathrm{s}}(\boldsymbol{r})=0$. The wetting properties can be probed either by measuring the contact angles of a drop placed on the surface, or more simply by measuring the three surface tensions of the liquid-solid $\left(\gamma_{\mathrm{SL}}\right)$, vapor-solid $\left(\gamma_{\mathrm{SV}}\right)$ and liquid-vapor $\left(\gamma_{\mathrm{LV}}\right)$ interfaces. The model presented here gives a direct access to the free-energy or the grandpotential (an advantage compared to molecular simulations); the surface tensions can thus be measured directly by considering a fluid confined between two structured walls as depicted in Fig 1-c. Provided the distance between the walls is large compared to the typical relaxation length of the density profile, the walls can be considered as independent. The relaxation length scale of the density profile is given by the interfacial thickness $w$, and we use a distance between the two walls of the order of $50 \mathrm{w}$, which ensures an accurate determination of the surface tensions.The liquid-solid [resp. vapor-solid] surface tension is measured by starting the simulation with a liquid [resp. vapor] phase between the walls. For planar interfaces this prescription is very efficient and the two surface tensions can be measured in the full range of variation of the interaction potential $V_{\mathrm{LJ}}$, which is done by varying $\epsilon$. From these two quantities, and the knowledge of $\gamma_{\mathrm{LV}}$, the contact angle with the planar surface can be calculated through the quantity $\Gamma=\frac{\gamma_{\mathrm{SV}}-\gamma_{\mathrm{SL}}}{\gamma_{\mathrm{LV}}} . \Gamma$ corresponds to $\cos \theta$ when $|\Gamma| \leq 1$ and is an increasing function of $\epsilon$ (attractions favor wetting of the liquid phase). We shall consider $\Gamma$ rather than $\epsilon$ in the discussions, since the contact angle is the experimentally relevant quantity. When the wall is not planar, and has a crenel shape as depicted in Fig 1 1 a, the situation is more complex since various states can be observed. We used in this case two different types of initial configurations: Wenzel states corresponding to a monophasic system, or Cassie states corresponding to a diphasic situation (see Fig 1-c). Of course, the equilibrium result should not depend on the initial state, this prescription gives us however a way to probe metastability in the system. The wetting properties of the structured wall can be measured as well by determining the effective surface tensions $\gamma_{\mathrm{SV}}^{\text {eff }}$ and $\gamma_{\mathrm{LV}}^{\text {eff }}$ for the equilibrated configurations, the variation of the corresponding wetting parameter $\Gamma^{\text {eff }}$ is plotted in Fig 2. This figure illustrates very well the Cassie-Wenzel transition between the imbibed and super-hydrophobic states [4]. Moreover, several important features can be noted from these results: first we observe a strong metastability, with the presence of superhydrophobic metastable states, and next, we observe an asymmetry in the wetting properties when we go from the non-wetting region to the wetting one. The macroscopic model describing the transition between Cassie and Wenzel states is compared to the numerical results in Fig.2. This model is quite accurate in

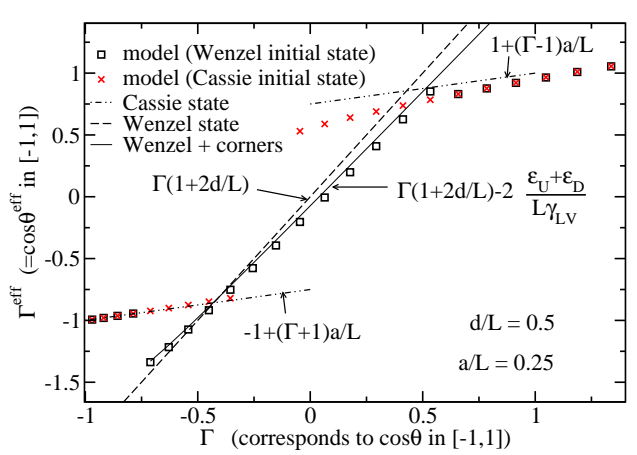

Figure 2: Wetting properties of a crenelated surface (see text for details).

the non-wetting region, which is not a surprise since we have the same geometry: the interfaces are planar at coexistence. In the wetting regime on the contrary, we observe discrepancies with the macroscopic theory. The difference comes from corner effects: the interaction potential between the fluid and the wall has particular values at the corners that generate a corner energy. These corner effects are mainly visible when a liquid phase fills the crenels (wetting situation), in gas phases the low value of the density leads to negligible contributions. If $\epsilon_{\mathrm{U}}$ and $\epsilon_{\mathrm{D}}$ are the excess free-energies in the liquid phase at the upper and the lower corners (see in Fig.1-c the points U and $\mathrm{D}$ ), the Wenzel theory can be modified as follows:

$$
\Gamma^{\mathrm{eff}}=\Gamma(1+2 d / L)-2 \frac{\epsilon_{\mathrm{U}}+\epsilon_{\mathrm{D}}}{L \gamma_{\mathrm{LV}}}
$$

where $\epsilon_{\mathrm{U}}$ and $\epsilon_{\mathrm{D}}$ are measured independently. This theory reproduces very well the effective contact angle (Fig 21). Please note that $\epsilon_{\mathrm{U}}$ and $\epsilon_{\mathrm{D}}$ are not constant, they vary slightly with $\cos \theta$, which is visible in Fig, 2 ,

We now turn to the dynamical properties of the surfaces. The dynamics of a fluid close to a textured wall can be accounted for using effective partial slip BC (3), where both the slip length $b$ and the hydrodynamic position of the wall $z_{\mathrm{s}}$ are unknown. For a smooth surface, it has been shown that the BC applies at about one molecular layer inside the fluid, for both no-slip and slip cases [17]. However for superhydrophobic surfaces, it is not clear where the $\mathrm{BC}$ should apply. In this work we therefore measured both $b$ and $z_{\mathrm{s}}$, by probing our system using both Couette and Poiseuille flows [17]. The geometry of the system corresponds to Fig 1 1 d), where the top wall is planar and the bottom one is structured. This hybrid geometry allows for the determination of the effective boundaries for both the planar and the textured interfaces at the same time. The figure corresponds to a Couette flow, for which the top wall moves with a velocity $\boldsymbol{U}$ perpendicular to the crenels, while the bottom wall is fixed. The Poiseuille situation corresponds to fixed walls, but a pressure gradient is applied. We shall inves- 


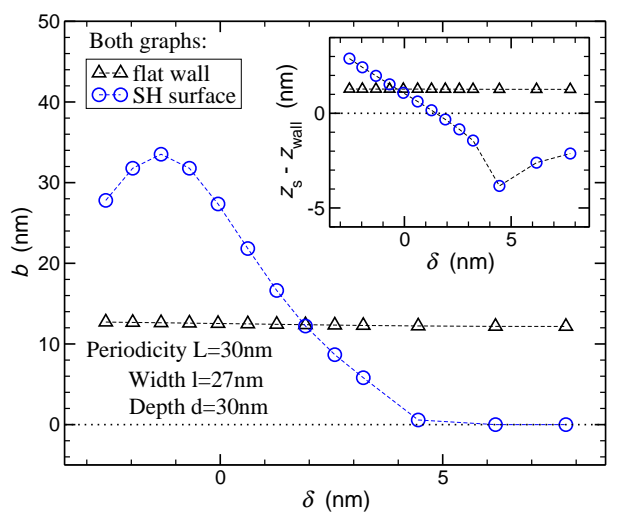

Figure 3: Evolution of the slip length $b$ with the recess $\delta$ of the meniscus inside the crenel, for a flow perpendicular to the crenel. Inset: Evolution of the shear plane position $z_{\mathrm{s}}$ as a function of $\delta$. For the SH surface, $z_{\mathrm{w}}$ is defined as the top of the crenels.

tigate the variation of both $b$ and $z_{\mathrm{s}}$ as a function of the liquid pressure. Rather than the pressure itself, we can equivalently fix the average density in the slab by using a conserved dynamics, and measure the pressure afterwards. We shall use $\delta$, the recess length of the liquid in the crenel (taken from the top of the crenels, as depicted in Fig 1-d), as the control parameter. $\delta$ can either be negative or positive. The slip length of the planar (bare) liquid-wall interface is taken to be around $13 \mathrm{~nm}$ at coexistence, and is observed to vary very weakly with pressure (Fig 3 ). On the contrary, the slip length of the structured wall varies in larger proportions, between $35 \mathrm{~nm}$ for the upper limit down to 0 for the lower one. Pressure has the effect to reduce slippage, and even to cancel it. The effective slip length crosses the bare one when $\delta_{\mathrm{c}} \simeq 2 \mathrm{~nm}$, and is lower above. This result is largely independent of the crenel depth since the depth we consider in the present simulation $(30 \mathrm{~nm})$ is large compared to the crossover length $\delta_{\mathrm{c}}$.

To conclude, the model presented here is very flexible and can account for complex BC. Although we focussed on crenelated walls, we can treat any type of geometry, 3D shapes such as posts or random surfaces, or conic shapes to model surfaces coated with nanotubes [18]. The dynamics on crenelated surfaces revealed the strong sensitivity of the effective slip length to the recess of the meniscus $\delta$ inside the crenels, cancelling slippage as soon as $\delta \simeq 2 \mathrm{~nm}$. This critical value increases with the periodicity $L$, although it remains a small fraction of it. However, increasing $L$ is not necessarily a good idea since the corresponding intrusion pressure will decrease. Although nanostructured surfaces have lower slip lengths than microstructured ones in general, the slip properties resist better to pressure. There is thus a compromise to find between slippage and pressure resistance.
[1] H. A. Stone, A. D. Stroock, and A. Ajdari, Annual Review Of Fluid Mechanics 36, 381 (2004).

[2] E. Lauga, M. P. Brenner, and H. A. Stone, Handbook of experimental fluid dynamics (Springer, 2005), chap. 15, to appear, cond-mat/0501557

[3] L. Bocquet and J. L. Barrat, Soft Matter 3, 685 (2007).

[4] A. Lafuma and D. Quere, Nat. Mater. 2, 457 (2003).

[5] C. Cottin-Bizonne, J. L. Barrat, L. Bocquet, and E. Charlaix, Nat. Mater. 2, 237 (2003).

[6] A. Dupuis and J. M. Yeomans, Langmuir 21, 2624 (2005).

[7] A. D. Stroock, S. K. Dertinger, G. M. Whitesides, and A. Ajdari, Anal. Chem. 74, 5306 (2002).

[8] C. Cottin-Bizonne, C. Barentin, E. Charlaix, L. Bocquet, and J. L. Barrat, European Physical Journal E 15, 427 (2004).

[9] N. V. Priezjev, A. A. Darhuber, and S. M. Troian, Physical Review E 71, 041608 (2005).
[10] M. Sbragaglia, R. Benzi, L. Biferale, S. Succi, and F. Toschi, Phys. Rev. Lett. 97, 204503 (2006).

[11] M. Sbragaglia and A. Prosperetti, Phys. Fluids 19, 043603 (2007).

[12] C. Kunert and J. Harting, Phys. Rev. Lett. 99, 176001 (2007).

[13] S. Chakraborty, Phys. Rev. Lett. 99, 094504 (2007).

[14] S.M. Allen and J.W. Cahn, Acta Met. 27, 1085 (1979).

[15] J.W. Cahn, Acta Met. 9, 795 (1961); J. W. Cahn and J. E. Hilliard, J. Chem. Phys 28, 258 (1957).

[16] J. S. Rowlinson and B. Widom, Molecular Theory of Capillarity (Oxford University Press, 1989).

[17] J. L. Barrat and L. Bocquet, Faraday Discuss. 112, 119 (1999).

[18] P. Joseph, C. Cottin-Bizonne, J. M. Benoit, C. Ybert, C. Journet, P. Tabeling and L. Bocquet, Phys. Rev. Lett. 97, 156104 (2006). 